\title{
TRIGGER SYSTEMS AT HADRON SUPER-COLLIDERS
}

\author{
NICK ELLIS \\ PH Department, CERN, 1211 Geneva 23, Switzerland
}

\begin{abstract}
This paper discusses the techniques used in state-of-the-art trigger systems, such as those currently under development for experiments at the Large Hadron Collider (LHC), and describes the new challenges that would have to be faced in a super-collider operating at very high energy and luminosity. In particular, it addresses the case of an upgraded LHC with very high luminosity and a short bunch-crossing interval.
\end{abstract}

\section{Introduction}

This paper starts with a review of some basic ideas about triggering in HighEnergy Physics (HEP) experiments before going on to describe how triggers are implemented in the general-purpose experiments at the Large Hadron Collider, LHC (i.e. in ATLAS [1] and CMS [2]). Issues to be addressed for experiments at a future hadron super-collider are identified, resulting from higher energy, higher luminosity, and reduced bunch-crossing $(\mathrm{BC})$ intervals, as well as from more complex detector systems (needed to cope with the experimental conditions at a super-collider). A number of areas are indicated where R\&D would be required.

\subsection{Some basic ideas in triggering for HEP experiments}

The role of the trigger in an HEP experiment is to make the online selection of particle collisions potentially containing interesting physics. High efficiency is needed for selecting processes of interest for physics analysis, and the efficiency should be precisely known. Furthermore, the selection should not have biases that affect physics results. A large reduction of rate from unwanted high-rate processes is needed in view of the limited capabilities of the data-acquisition system (DAQ) and also offline computing facilities (both processing power and storage). Backgrounds may be instrumental (e.g. due to beam losses in the vicinity of the detector systems) or from high-rate physics processes that are not relevant for analysis. Another essential requirement is that the trigger system must be affordable, which limits complexity of algorithms that can be used. It is worth noting that it is not easy to achieve all the above requirements simultaneously! 
Modern HEP experiments use multi-level triggers that provide rapid rejection of high-rate backgrounds without incurring (much) dead-time ${ }^{\mathrm{a}}$, together with high overall rejection to reduce the event and data rates to mass storage to manageable levels. The fast first-level trigger (LVL1), generally implemented using custom electronics, needs high efficiency, but its rejection power can be comparatively modest. Here, short latency ${ }^{b}$ is essential since information from all (up to $O\left(10^{8}\right)$ already at LHC) detector channels needs to be buffered (often ondetector) pending the LVL1 result. Progressive reduction in rate after each stage of selection allows the use of more and more complex algorithms at affordable cost. The final stages of selection, running on computer farms, can use comparatively very complex (and hence slow) algorithms to achieve the required overall rejection power.

\subsection{Physics considerations}

It is interesting to consider the trigger requirements from a physics perspective. Typically, trigger systems select events according to a "trigger menu", i.e. a list of selection criteria. An "event" is selected by the trigger if one or more of the selection criteria are met. Note that in this paper the term "event" is used to mean the record of the activity in a given $\mathrm{BC}$ - typically an event at LHC contains many proton-proton interactions - this implies that the LVL1 trigger has to identify the BC of interest.

Different selection criteria may correspond to different signatures for the same physics process since redundant selections lead to high selection efficiency and allow the efficiency of the trigger to be measured from the data. Different criteria may also reflect the wish to concurrently select events for a wide range of physics studies. HEP "experiments" - especially those with large generalpurpose "detectors" (i.e. detector systems) — are really experimental facilities.

It is very important to remember that events rejected by the trigger are lost forever! In contrast to offline processing and physics analysis, there is no possibility of a second chance!

Discovery physics is the main emphasis for ATLAS and CMS at LHC, and in general for future hadron-super-collider experiments. There is a huge range of predicted new physics processes with diverse signatures, and very low signal

\footnotetext{
a The term "dead-time" refers to periods when interesting interactions cannot be selected, for example because the necessary Trigger/DAQ resources are unavailable.

b The term "latency" means the time taken to form and distribute the trigger decision.
} 
rates are expected in some cases (as little as $10^{-16}$ of total rate). However, one should also try to be sensitive to new physics that has not been predicted!

A super-collider would operate after LHC and therefore be relevant for the discovery of very high-mass objects (i.e. those beyond the reach of LHC). Supercollider experiments would also perform precision measurements of lower-mass objects already discovered at LHC.

Figure 1 shows examples of cross-sections and rates at hadron colliders. There is, of course, a huge rate of Standard Model (SM) physics backgrounds. The total rate of proton-proton collisions is up to $10^{9} \mathrm{~Hz}$ at LHC, and even more for future hadron super-colliders. For reference, ATLAS and CMS each aim for event storage rates of $O(100) \mathrm{Hz}$, i.e. $O\left(10^{-7}\right)$ of the total interaction rate, with a typical event size of $\sim 1$ MByte.

The overall rates (and hence pile-up ${ }^{c}$ and detector occupancy) obviously increase with luminosity. The total cross-section grows only slowly with energy, while cross-sections for new physics and also for SM backgrounds (jets, W, Z) increase more rapidly with energy. Much lower rates are predicted for instrumental backgrounds such as beam-gas interactions.
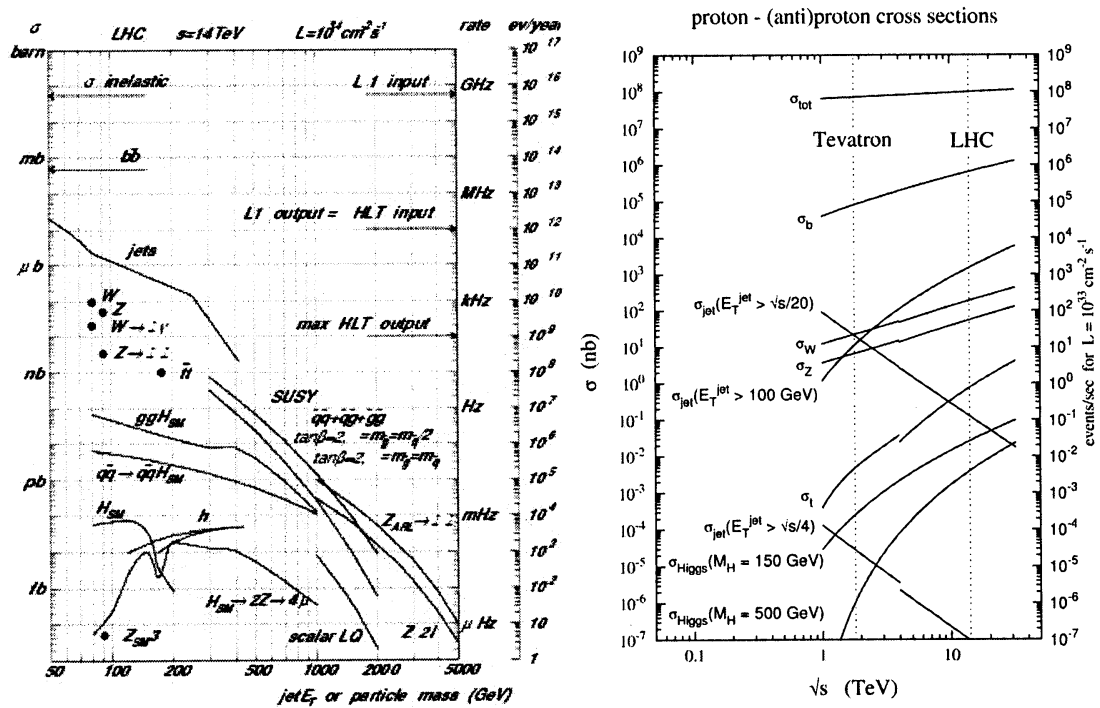

Figure 1: Examples of cross-sections and rates at hadron colliders. The left-hand figure shows rates under the design conditions at LHC as a function of particle mass or jet transverse energy. The right-hand figure shows the dependence of rates on centre-of-mass energy.

\footnotetext{
${ }^{c}$ Pile-up refers to the superposition of information from multiple interactions in the same $\mathrm{BC}$, which can cause confusion for the reconstruction of the interaction of interest.
} 
The trigger will have to retain as many as possible of the events of interest for the diverse physics programmes of super-collider experiments, including: Higgs searches (SM and beyond, e.g. $\mathrm{H} \rightarrow \mathrm{ZZ} \rightarrow$ leptons (e or $\mu$ ), $\mathrm{H} \rightarrow \gamma \gamma$, and also $\mathrm{H} \rightarrow \tau \tau, \mathrm{H} \rightarrow b \bar{b}$ ); SUSY searches (e.g. producing jets and missing transverse energy); searches for other new physics for which one will use inclusive triggers that one hopes will be sensitive to unpredicted new physics; studies of SM processes which must be understood as backgrounds to new physics, e.g. production of $\mathrm{W}$ and $\mathrm{Z}$ bosons, top and beauty quarks.

In contrast to the particles produced in typical proton-proton collisions (typical hadron transverse momentum, $p_{\mathrm{T}} \sim 1 \mathrm{GeV}$ ), the products of new physics are expected to have large $p_{\mathrm{T}}$. For example, those produced in the decay of a new heavy particle such as a Higgs boson with mass, $m \sim 100 \mathrm{GeV}$, typically have $p_{\mathrm{T}} \sim 50 \mathrm{GeV}$. Representative examples of LVL1 thresholds for ATLAS and $\mathrm{CMS}$ at $10^{34} \mathrm{~cm}^{-2} \mathrm{~s}^{-1}$ luminosity are: single-muon $p_{\mathrm{T}}>20 \mathrm{GeV}$ (rate $\sim 10 \mathrm{kHz}$ ); pair of muons each with $p_{\mathrm{T}}>6 \mathrm{GeV}$ (rate $\sim 1 \mathrm{kHz}$ ); single e $/ \gamma p_{\mathrm{T}}>30 \mathrm{GeV}$ (rate $\sim 20 \mathrm{kHz}$ ); pair of e/ $\gamma$ each with $p_{\mathrm{T}}>20 \mathrm{GeV}$ (rate $\sim 5 \mathrm{kHz}$ ); single jet $p_{\mathrm{T}}>300 \mathrm{GeV}($ rate $\sim 200 \mathrm{~Hz})$; jet $p_{\mathrm{T}}>100 \mathrm{GeV}$ in coincidence with missing $-p_{\mathrm{T}}$ $>100 \mathrm{GeV}\left(\right.$ rate $\sim 500 \mathrm{~Hz}$ ); four or more jets $p_{\mathrm{T}}>100 \mathrm{GeV}($ rate $\sim 200 \mathrm{~Hz}$ ). More details on the LVL1 triggers for ATLAS and CMS can be found in Refs. [3] and [4] respectively. The corresponding High-Level Triggers (HLT) are described in Refs. [5] and [6]. A general pedagogic discussion of Trigger/DAQ systems is given in Ref. [7] upon which much of the background material presented by the speaker was based.

\section{LHC and hadron super-colliders}

Some of the key design parameters of the LHC affecting the design of trigger systems are the centre-of-mass energy $(14 \mathrm{TeV})$, the luminosity $\left(10^{34} \mathrm{~cm}^{-2} \mathrm{~s}^{-1}\right)$, the $\mathrm{BC}$ interval $(25 \mathrm{~ns})$ and the anticipated average number of interactions per BC $(\sim 25)^{d}$. These parameters already give a very challenging environment for making the trigger.

The conditions at a future hadron collider will certainly be even more daunting for the development of trigger systems. Higher energy, while very interesting in the longer term from the point of view of accessing physics at high mass scales, would require a completely new machine. Higher luminosity, on the other hand, could be achieved via an upgrade of the LHC machine and the ATLAS and CMS experiments. Such a Super-LHC (SLHC) might reach a luminosity as high as $10^{35} \mathrm{~cm}^{-2} \mathrm{~s}^{-1}$, as discussed in Ref. [8].

\footnotetext{
${ }^{\mathrm{d}}$ Note that this situation in a hadron collider is very different to that in $\mathrm{e}^{+} \mathrm{e}^{-}$ colliders where the interaction rate is tiny compared to the $\mathrm{BC}$ rate.
} 
The number of interactions per $\mathrm{BC}$ at a future very high-luminosity hadron collider could be limited by using a BC period shorter than $25 \mathrm{~ns}-$ e.g. $12.5 \mathrm{~ns}$ is considered for SLHC (which still implies an anticipated average of about 125 interactions per BC, c.f. $\sim 25$ at $\mathrm{LHC}$ ).

The BC period is a parameter of the machine that profoundly affects the LVL1 trigger design. At least at LHC, the LVL1 trigger must uniquely identify the $\mathrm{BC}$ of interest - this simplifies detector front-end electronics and readout, and also minimizes the event size.

\section{First-level triggers at LHC}

The LVL1 trigger has to deliver a new decision every $\mathrm{BC}$, while the trigger latency (typically $\sim$ few $\mu$ s) is much longer than the $\mathrm{BC}$ period. This means that LVL1 must concurrently process many events. This is generally achieved by "pipelining" the processing in custom trigger processors built using modern digital electronics, breaking the processing down into a series of steps, each of which can be performed within a single BC period. Many operations can be performed in parallel by having separate processing logic for each one. Note that the latency of the trigger is fixed, determined by the number of steps in the calculation plus the time taken to move signals and data to and from the components of the trigger system (signals have to pass from the detector to the trigger electronics and back, with a round-trip distance of about $200 \mathrm{~m}$ corresponding to $\sim 1 \mu$ s delay). See Refs. [3] and [4] for details of the ATLAS and CMS LVL1 trigger systems.

The data processing technologies used in modern LVL1 trigger systems are typically FPGAs (Field Programmable Gate Arrays) and ASICs (ApplicationSpecific Integrated Circuits). FPGAs (and other programmable devices) now play a very important role since they have large numbers of logic gates and many I/O pins available, they operate at $40 \mathrm{MHz}$ and above, and hence have performance sufficient for implementing many trigger algorithms. FPGAs offer huge flexibility and the possibility to modify algorithms as well as parameters of algorithms once experiments start running. ASICs are used for some applications since they are more cost effective in some cases (e.g. large number of devices required), they can offer higher speed performance than FPGAs, and they can have better radiation tolerance and lower power consumption for on-detector applications.

Data-movement is a major issue in LVL1 trigger systems (see Fig. 2). At LHC the LVL1 triggers use quite fine-grained information from the detectors (although not as fine as in the HLT and offline). Generally data need to be fanned-out between processing elements (e.g. environment information for 
calculating cluster-shape parameters in the calorimeters). Then the information from different parts of the detector is combined through a tree of pipelined processing stages, ultimately giving a single-bit (yes/no) output for each BC.

Concerning the implementation, high-speed serial data links (electrical and optical) are widely used, e.g. comparatively inexpensive and low-power LVDS links for electrical transmission at $\sim 400 \mathrm{Mbit} / \mathrm{s}$ over distances up to $\sim 10 \mathrm{~m}$, or $\mathrm{Gbit} / \mathrm{s}$ devices using optical transmission for longer distances. Very high-density custom backplanes are used to share information between processing elements, with large pin-counts (up to $\sim 800$ connections per $9 \mathrm{U}$ board), and high data rates per (point-to-point) connection, e.g. $\sim 160 \mathrm{Mbit} / \mathrm{s}$. Note that data are multiplexed to speeds beyond $40 \mathrm{Mbit} / \mathrm{s}$ to reduce connectivity problems to a level that can be managed. Typically the processors use large printed-circuit boards since it is easier to handle interconnections on-board than between boards.

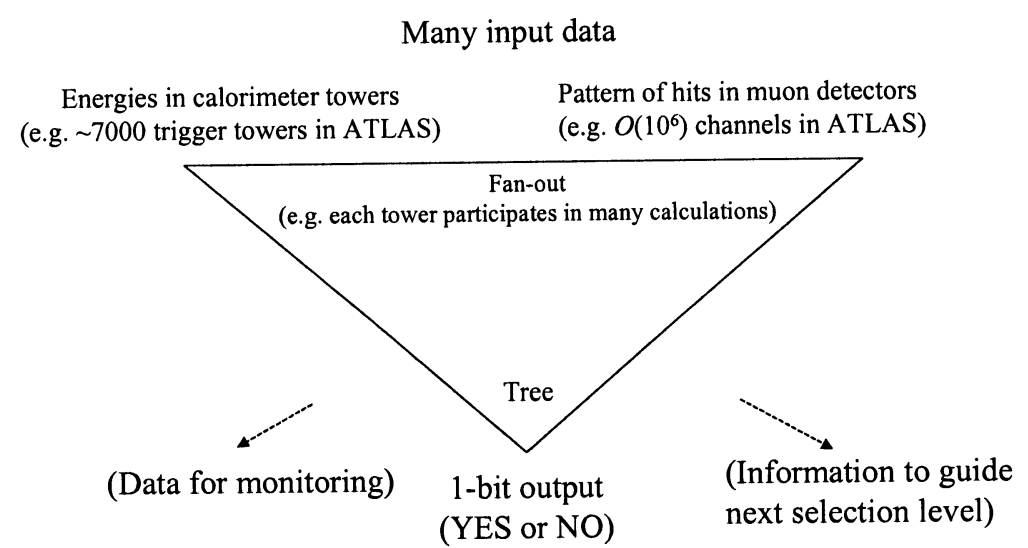

Figure 2: Data flow in LVL1 trigger systems.

\subsection{Case study: ATLAS first-level calorimeter trigger}

The ATLAS calorimeter trigger can be used as an example to illustrate some general issues related to LVL1 triggers at LHC. It is organized as follows (see Fig. 3, left). Analogue electronics on the detector sums the calorimeter raw signals to form "trigger towers", the signals for which are sent electrically to an underground electronics "counting" room. The signals are received and digitized, and the digital data are processed to measure transverse-energy, $E_{\mathrm{T}}$ per tower for each $\mathrm{BC}$, giving an $E_{\mathrm{T}}$ matrix for each of the electromagnetic and hadronic calorimeters. The tower data are transmitted to the Cluster Processor (CP, 4 crates) and Jet-Energy Processor (JEP, 2 crates), fanning-out those values needed in more than one crate (giving motivation for a very compact design of processor). Within the CP and JEP crates, values need to be fanned-out between 
electronic modules, and between processing elements on the modules. Connectivity and data-movement issues drive the design.

The raw calorimeter signals extend over many $\mathrm{BCs}$. One therefore needs to combine information from a sequence of measurements to estimate the energy and identify the $\mathrm{BC}$ where the energy was deposited. As shown in the right-hand part of Fig. 3, this is achieved using a Finite Impulse Response filter that feeds a look-up table (LUT) to convert the data to transverse-energy units and a peak finder to determine the $\mathrm{BC}$ where energy was deposited. Special attention is given to issues of signal distortion for very large pulses since one must not lose the most interesting physics! An ASIC incorporates the above and includes datacompression (so-called BC multiplexing [3]) on the outputs to the $\mathrm{CP}$.
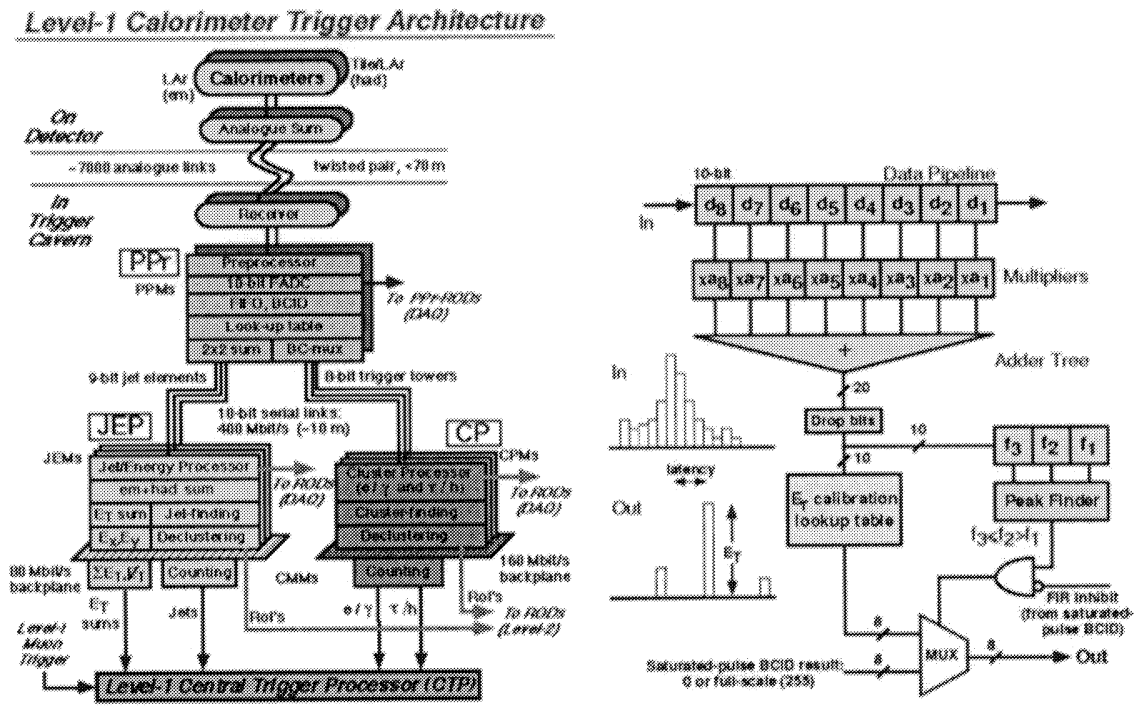

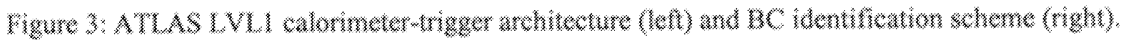

As an illustration, the ATLAS electron/photon trigger is based on $4 \times 4$ "overlapping, sliding" windows of trigger towers. Each trigger tower spans $0.1 \times 0.1$ in $\eta \times \phi$ ( $\eta$ pseudo-rapidity, $\phi$ azimuth), and there are $\sim 3500$ such towers in each of the electromagnetic and hadronic calorimeters. The algorithm (see Fig. 4) is performed in parallel in -3500 windows every $25 \mathrm{~ns}$; each tower participates in calculations for 16 windows. The associated issue of connectivity is a driving factor in the trigger design.

The array of $E_{T}$ values computed in the Pre-processor (PPr) has to be transmitted to the CP every $25 \mathrm{~ns}$. This is done using digital electrical (LVDS) links to the CP modules, with $\sim 5000$ links at 400 Mbit's for a total of $\sim 2000$ Gbit's (equivalent to $O(100 \mathrm{M})$ voice calls) after using $\mathrm{BC}$-multiplexing 
compression. The data are converted to $160 \mathrm{Mbiv}$ single-ended signals on the CP modules before faming -out data to neighbouring modules over very highw density custom back-planes ( 800 pins per slot in a 90 crate, 160 Mbivs pointto-point). Data are amed rout to eight large processing FPGAs in each $\mathrm{CP}$ module; the FPGAs receive data at $160 \mathrm{Mbiv}$.

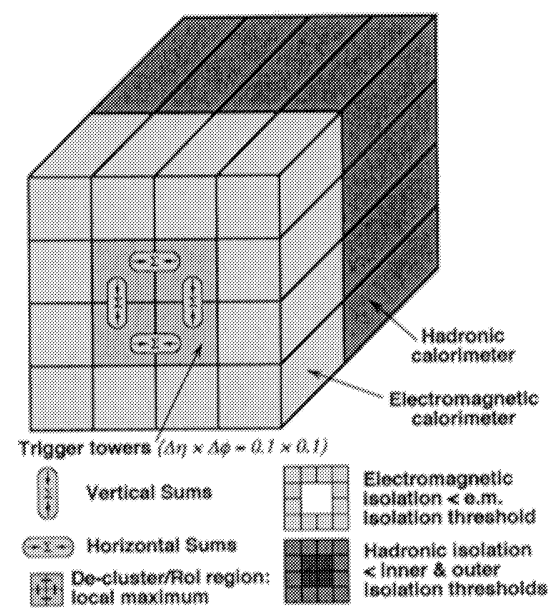

Figure A ATUAS LVL cectron/photon trigger algorithm.

The electron/photon (together with the tauhadron) algorithm is implemented in FPAAs. This has only become feasible with recent advances in FPCA technology since very large and fast devices are needed. Each FPGA handles $4 \times 2$ windows, and so needs data from $7 \times 5 \times 2$ towers $(\eta \times 6 \times(\mathrm{E} / \mathrm{H})$. The algorithm is described in a language (VWDL) that can be converted into the FPGA configuration hle, giving flexibitity to adapt algoritms in the light of experience. Parameters of the algorithms can be changed easily, $e . g$. cluster $E_{r}$ turesholds are held in registers that can be programmed without reconfiguring the $\mathrm{gPGAs}$.

\section{Tirst-level triggers for a hadron super-collider}

In the following, various issues for a future hadron super-collider are discussed, taking the conditions at $L H C$ as a reference.

\subsection{Higher luminosity}

Higher luminosity obviously results in increased rates for all processes. The increased rates for high $p_{T}$ processes can be compensated by rasing the $p_{2}$ thresholds, and/or enhancing the rate capabilites of DAQ, HLT and Omine computing, and/or improving the algorithms to get more background-rejection 
power by using more detailed information. Note that increased pile-up degrades the performance of the algorithms, e.g. isolation requirements become less effective at separating signal and background, which can be compensated by raising the $p_{\mathrm{T}}$ thresholds further and/or reducing the $\mathrm{BC}$ interval (to get fewer interactions per $\mathrm{BC}$ for fixed interaction rate). Finer detector granularity could also be used to reduce the occupancy at fixed luminosity.

Another consequence of higher luminosity is increased radiation levels. This puts stronger requirements for shielding and/or radiation tolerance of on-detector trigger electronics. There are possible implications for personnel access to underground counting rooms in case of SLHC. Background hits in the muon detectors, induced by the radiation in the detector caverns, are potentially a problem for muon triggers (fake tracks from accidental coincidences).

\subsection{Higher energy}

Increasing the collision energy (see Fig. 1) has less impact on trigger design than increasing the luminosity. The total cross-section grows slowly with energy, so the increase in pile-up is relatively modest; the increase in radiation levels in the caverns is also comparatively modest. However, shielding in the forward regions would be important given the total power dissipation from interaction products.

Higher collision energy results in increased rates for high $-p_{\mathrm{T}}$ processes (both signal and background). This can be compensated by raising the $p_{\mathrm{T}}$ thresholds, and/or enhancing the rate capabilities of DAQ, HLT and Offline computing, and/or improving the algorithms to get more background-rejection power by using more detailed information.

\subsection{Shorter bunch-crossing interval}

A shorter BC interval would have several important consequences for the LVL1 trigger. Processing more data per unit time is feasible given continuing advances in microelectronics (e.g. FPGAs) that allow faster clock speeds. However, the data-movement aspects of the LVL1 triggers are already very challenging at LHC, e.g. very high-density and very high-speed backplanes, and R\&D would be required in this area addressing data-movement technologies and datacompression techniques. Another issue is that of "timing-in" the experiment. Fine-tuning of timing in LHC experiments relies on resolving the bunch structure in the trigger and front-end electronics. This model might not be viable for $\mathrm{BC}$ intervals less than $\sim 10 \mathrm{~ns}$.

At this point it is interesting to come back to the case study of the ATLAS calorimeter trigger discussed in Section 3.1. For the parameters of SLHC with a $\mathrm{BC}$ period of $12.5 \mathrm{~ns}$, digitization of the raw calorimeter signals should not be a 
problem given the availability of $80 \mathrm{MHz}$ fast ADCs. Similarly, digital signal processing clocked at $80 \mathrm{MHz}$ is feasible (indeed parts of the trigger systems, e.g. in the CMS calorimeter trigger, are already clocked at multiples of $40 \mathrm{MHz}$ ). However, data-movement aspects would certainly require substantial R\&D investigating faster connections and more aggressive data-compression schemes.

Substantially shorter BC intervals would have important consequences for the LVL1 trigger and detector electronics. At $25 \mathrm{~ns}$ (probably also at $12.5 \mathrm{~ns}$ ), the detectors and LVL1 can resolve the BC that contained the interaction of interest. The detector signals are digitized (or analogue-buffered) once per $\mathrm{BC}$ and the time of the interaction is known at $\sim 1 \mathrm{~ns}$ precision from the $\mathrm{BC}$ time. Operation with quasi-continuous beam would require a significantly different approach. One could clock the LVL1 trigger and front-end electronics with a frequency less than the $\mathrm{BC}$ frequency of machine (e.g. $\mathrm{BC} \div \mathrm{N}$ or using a freerunning clock). However, many issues would need to be addressed. In particular, the need to handle timing ambiguities, since in an environment with a high level of pile-up "event" no longer has clear meaning. Clearly, ambiguities would not be easy to sort out on the timescale of the LVL1 trigger $(\sim$ few $\mu$ s $)-$ e.g. $E_{\mathrm{T}}$ values in different towers forming a cluster would need to be matched in time as well as in space (cluster threshold, isolation thresholds, etc). The LVL1 trigger (and also detector electronics) for such a machine would clearly require extensive R\&D.

\subsection{More granular detectors}

The use of finer detector granularity in the LVL1 trigger would offer the advantage of lower occupancy in the presence of pile-up and potentially better background-rejection power, e.g. for the calorimeter-based triggers. In the case of the muon trigger, it would allow more precise $p_{\mathrm{T}}$ measurement for a fixed magnet configuration (i.e. better spatial resolution), but at the expense of having to move and process more data at LVL1.

Reading out more granular detectors would increase the amount of data to be stored during the LVL1 latency (giving motivation to keep latency as short as possible) and to be moved to the HLT and DAQ (giving motivation not to enlarge time-frames for readout more than necessary). Extended use of data compression techniques could offer scope to limit the increase in the event size.

\subsection{First-level trigger menu for $S L H C$}

A very rough assessment has been made [8] of LVL1 trigger thresholds for SLHC at $10^{35} \mathrm{~cm}^{-2} \mathrm{~s}^{-1}$ luminosity. Some illustrative examples are: single-muon $p_{\mathrm{T}}$ $>30 \mathrm{GeV}\left(\right.$ rate $\sim 25 \mathrm{kHz}$ ); single e $/ \gamma p_{\mathrm{T}}>55 \mathrm{GeV}$ (rate $\sim 20 \mathrm{kHz}$ ); pair of e $/ \gamma$ 
each with $p_{2}>30 \mathrm{GeV}($ rate $-5 \mathrm{kHz}) ;$ single jet $p_{\mathrm{T}}>350 \mathrm{GeV}($ rate $-1 \mathrm{kHz}) ;$ jet $p_{\psi}>150$ GeV in coincidence with missing $p_{\mathrm{T}}>80 \mathrm{GeV}($ rate $\sim 1-2 \mathrm{kHz}$ ). The selection would be less inclusive than at LHC, but very high thesholds should be acceptable for SLHC discovery physics where one would be looking for objects and interactions at very high mass scales. More exclusive selections could be used for precision measurements of particles already discovered at LHC.

\section{Migh-level triggers and DAQ at LHC}

In the LHC experiments, data are transferred to large buffer memonies after a LVILI accept (see Wig, 5). In normal operation, the subsequent stages should not introduce further dead-time. The data rates at the $\mathrm{HTT} / \mathrm{DAQ}$ input are stll massive ( $\sim$ MByte event size after data compression at $-100 \mathrm{kHz}$ event rate, for $-100 \mathrm{GByte} / \mathrm{s}$ data rate). This is far beyond the capacity of the bus-based event building of e.g. LEP. Network-based event building is therefore used to avoid bandwidth bottlenecks. Data are stored in Read oul Systems untl they have been transferred to the Filter Systems (associated with HLT processing), or until the event is rejected. No node in the system sees the full data rate - each Read-out System covers only a part of the detector - each Fiter System deals with only a fraction of the events.

The massive data rate after LVL1 poses problems even for network-based event building - different solutions are being adopted to address this. For example. in CMS the event building is factorized into a number of slices each of which sees only a fraction of the rate (see Fig. 6). This requires large total network bandwidth, but avoids the need for a single, very-large network switch. An additional advantage of the "sliced" event-building approach is that not all slices need to be implemented intially given that there are funding limitations.

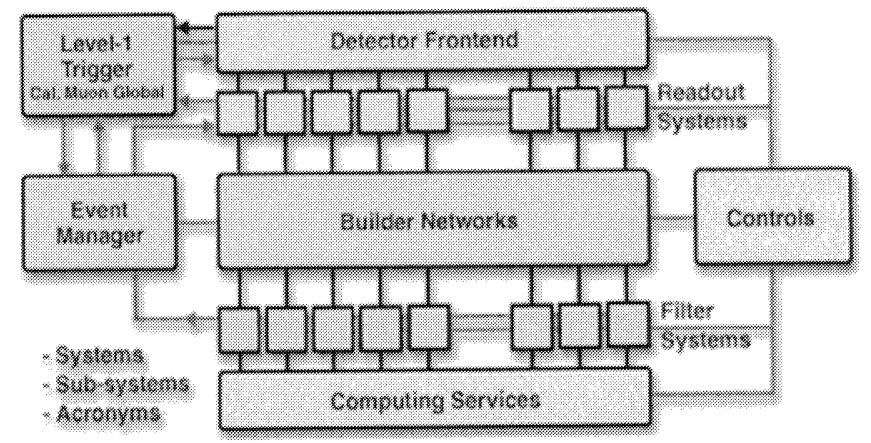

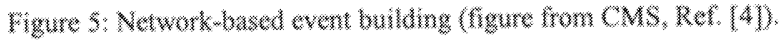




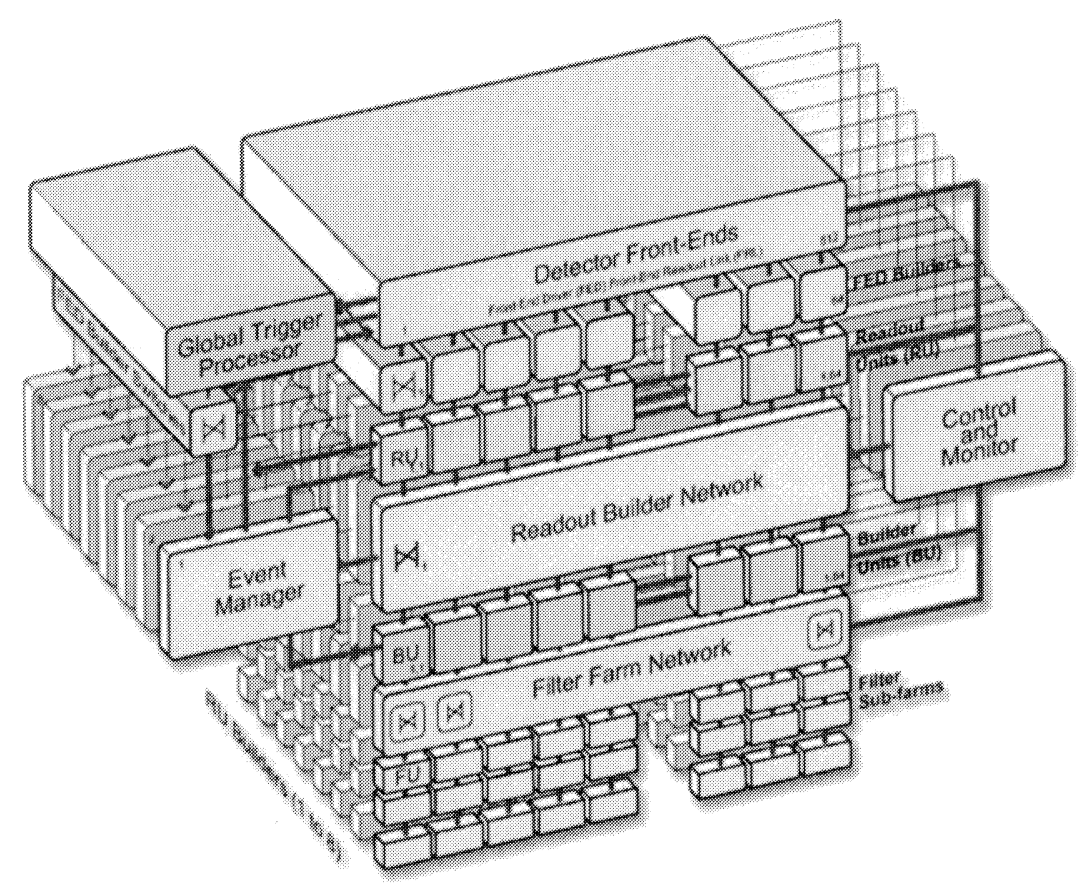

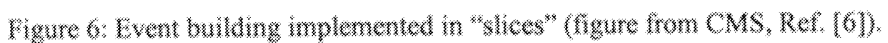

In ATLAS, the Regionmof-Interest (Rol) mechanism is used to access the data selectively - only data needed for second-level trigger (LVL2) processing are requested. This reduces by a substantial factor the amount of data that needs to be moved from the Read -out Systems to the processors, but implies relatively complicated mechanisms to serve the data selectvely. The basic idea of the Rol mechanism is that LVL1 indicates the geographical location of candidate objects, e.g. the wo muon candidates in the event shown in Fig. 7, LVL2 then only accesses data from these Rols. Within the Rols the selection process is performed sequentially. Data are accessed by LVL 2 initually only from a subset of detectors (e,g. muon spectrometer only), other detectors being used only for Rols that pass the initial selection. Many events are rejected without accessing the other detectors at all, giving a further reduction in total data transfer rate. The ATLAS HLTD AO system is Ilustrated in Fig. 8 . 


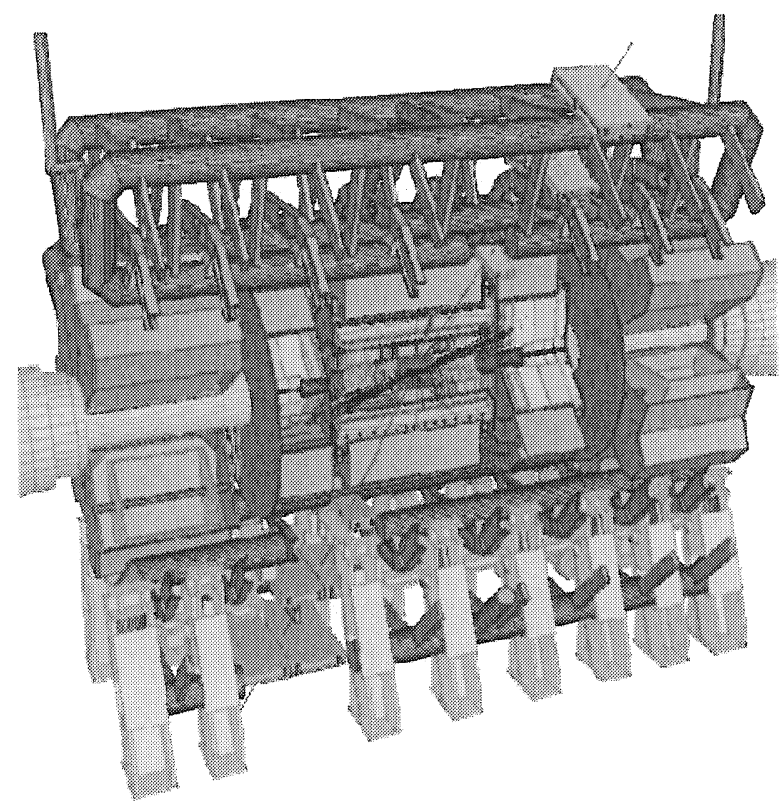

Figure 7: Example of a dimuon event in ATLAS - note that only a small fraction of the detector volume contains information relevant to the selection based on the two muons.

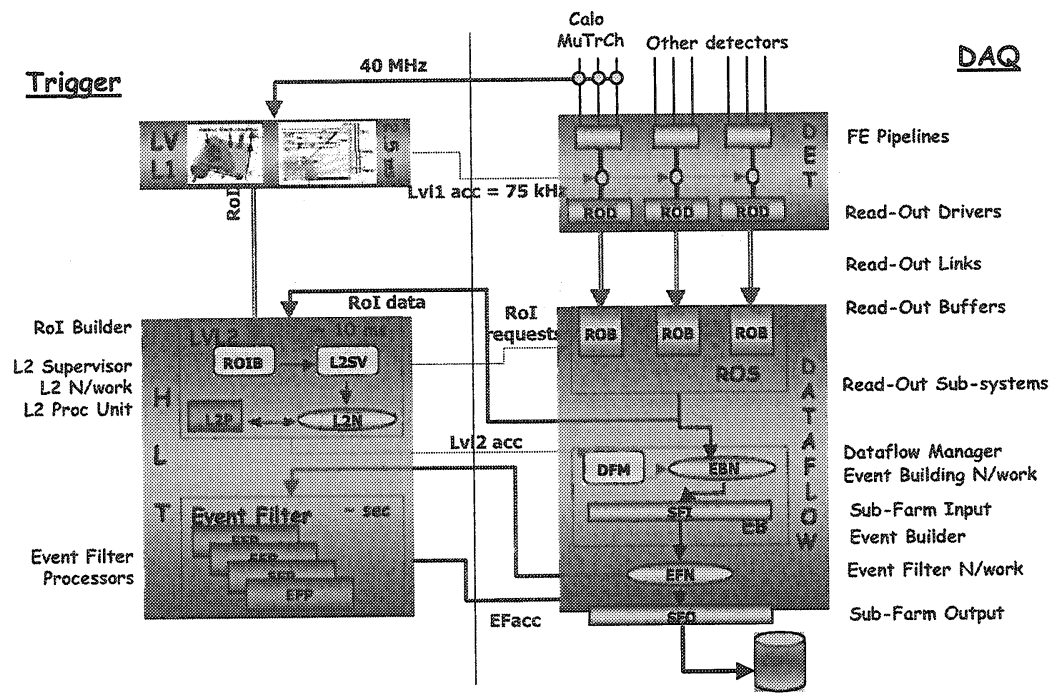

Figure 8: ATLAS HLT/DAQ system (from Ref. [9]).

\section{High-level trigger for a hadron super-collider}

The computer industry provides the technologies that will be used to build much of the HLT and DAQ systems at LHC. Computer networks and switches already 
provide high data-movement performance at affordable cost. PCs offer exceptional value for money in processing power, and high-speed network interfaces are standard items (e.g. Ethernet at $1 \mathrm{Gbit} / \mathrm{s}$ ). Continued improvements in these technologies, driven by mass-market applications, should provide the tools to implement the HLT for experiments at a hadron super-collider, as discussed in Ref. [10].

It is worth noting that the concepts used in ATLAS and CMS could be combined, i.e. sliced readout combined with the RoI mechanism and sequential data access, processing and selection, as illustrated in Fig. 9.

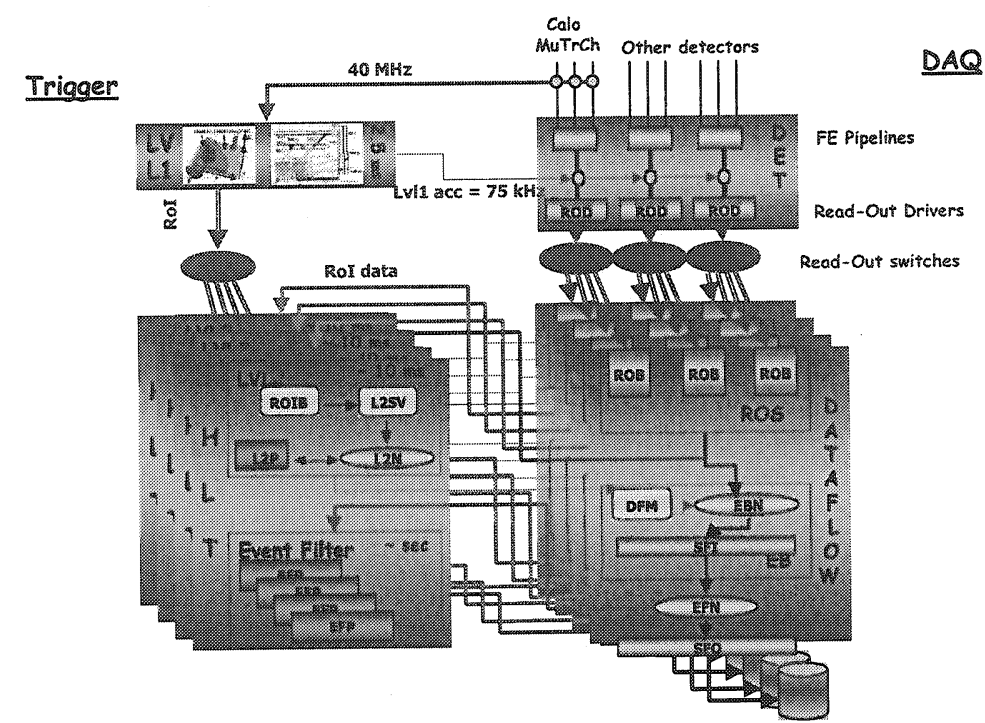

Figure 9: Illustration of a HLT/DAQ system incorporating slicing together with the Rol mechanism and sequential data access.

\section{Conclusions}

In conclusion, the following is a list of some key issues that require study for trigger systems at hadron-Super-Colliders:

- Improved LVL1 trigger algorithms and architectures (more complex algorithms, using more information) to achieve more rejection power for fixed efficiency (and at affordable cost!).

- Data movement in the LVL1 trigger to handle more data in case of a reduced $\mathrm{BC}$ period and/or increased detector granularity; this should include data-transfer technologies and data-compression techniques.

- Implications of very short $\mathrm{BC}$ intervals that could have a major impact on the LVL1 trigger (and detector electronics) design. 
- Improved HLT/DAQ architectures capable of supporting large data bandwidth and processing power to cope with increased LVL1 rates and/or increased event-size, e.g. combine slicing, RoI and sequential-processing concepts.

\section{Acknowledgements}

The author thanks members of the ATLAS and CMS experiments for their help in preparing this paper, especially Sergio Cittolin, Eric Eisenhandler, Fabiola Gianotti, Livio Mapelli, Peter Sharp, Wesley Smith, Paris Sphicas and Alan Watson. He also acknowledges his fellow members of the study group on the Physics Potential and Experimental Challenges of the LHC Luminosity Upgrade (see Ref. [8] upon which many of the ideas presented here are based). The author thanks Pierre Vande Vyvre for useful discussions in preparing his slides for the Workshop.

The author would like to express his gratitude to Professor Zichichi, as well as to the Workshop Directors, Eugenio Nappi and Jacques Seguinot, for the excellent hospitality, facilities and workshop organization in Erice. He very much appreciated the wonderful environment and atmosphere that stimulated many fascinating discussions between the participants.

\section{References}

1. ATLAS detector and physics performance Technical Design Report, CERN/LHCC/99-14.

2. CMS Technical Proposal, CERN/LHCC/94-38.

3. ATLAS first-level trigger Technical Design Report, CERN/LHCC/98-14.

4. CMS level-1 trigger Technical Design Report, CERN/LHCC/2000-038.

5. ATLAS high-level trigger, data acquisition and controls Technical Design Report, CERN/LHCC/2003-022.

6. CMS data acquisition and high-level trigger Technical Design Report, CERN/LHCC/2002-26.

7. N. Ellis, "Trigger and data acquisition", in Proc. 2001 CERN-CLAF School of High-Energy Physics, CERN-2003-003, pp. 253-276.

8. F. Gianotti et al., "Physics potential and experimental challenges of the LHC luminosity upgrade", hep-ph/0204087.

9. L. Mapelli, talk presented at Open Session of LHCC, CERN, 24 September 2003, available from: http://agenda.cern.ch/fullAgenda.php?ida $=\mathrm{a} 032160$.

10. P. Vande Vyre, "Data acquisition and mass storage", in these proceedings. 